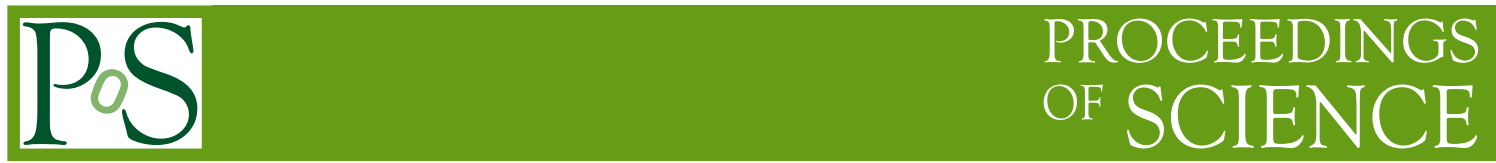

\title{
Measurements of Heavy Cosmic Ray Nuclei Fluxes with CALET
}

\author{
Yosui Akaike* \\ Astrophysics Science Division, NASA Goddard Space Flight Center, Greenbelt, MD 20771, USA \\ Center for Research and Exploration in Space Science \& Technology II \\ Center for Space Science and Technology, University of Maryland Baltimore County, Baltimore, \\ MD 21250, USA \\ E-mail: yosui.akaike@nasa.gov
}

\section{for the CALET Collaboration ${ }^{\dagger}$}

The CALorimetric Electron Telescope (CALET) has been measuring high-energy cosmic rays on the International Space Station since October 2015. One of the scientific objectives is the precise measurements of the boron-to-carbon flux ratio and the energy spectra of nuclei to investigate the cosmic-ray acceleration and propagation in the Galaxy. The instrument has capabilities to identify individual nuclei elements up through Iron with excellent charge resolution and cover the wide energy range from $10 \mathrm{GeV}$ to a PeV. In this paper, we will present the details about the analysis of nuclei measurements and preliminary results of boron-to-carbon flux ratio and energy spectra of heavy nuclei components up to Iron based on three years of operations.

36th International Cosmic Ray Conference -ICRC2019-

July 24th - August 1st, 2019

Madison, WI, U.S.A.

\footnotetext{
* Speaker.

${ }^{\dagger}$ for collaboration list see Pos(ICRC2019)1177
} 


\section{Introduction}

Direct measurement of cosmic-ray energy spectra and their flux ratio is essential to understand their origin, acceleration and propagation in the Galaxy. The acceleration in supernova remnants and diffusive propagation in the galactic magnetic fields are commonly accepted as a plausible scenario for the galactic cosmic-ray origins, however, the details are unknown. In particular, recent observations have indicated the presence of the hardening at a few hundreds of $\mathrm{GeV} / \mathrm{n}$ in the energy spectra of proton [1-3] and helium [1,4] as well as heavy nuclei $[5,6]$ that are unexpected features with respect to the general theoretical predictions. Due to the fact that the secondary species are produced during the propagation of primary cosmic rays, secondary-to-primary ratios such as B/C ratio are the key to clarify the propagation mechanism of cosmic rays in the Galaxy. Precise measurements of the cosmic-ray fluxes and the extension of the measurement up to the $\mathrm{TeV}$ scale provide important new data to test the theoretical models.

CALET is a cosmic-ray experiment on the International Space Station [7,8], and has been collecting science data since mid-October 2015. The detector is optimized to measure the electron spectrum in the trans $\mathrm{TeV}$ region and the results have been reported $[9,10]$. CALET also measures the energy spectra and elemental composition of cosmic-ray nuclei from proton to iron in the range from a few tens of $\mathrm{GeV}$ to the PeV scale. The features of the CALET instrument include a very good energy resolution provided by its thick calorimeter and excellent charge resolution and robust track identification based on the segmented scintillator paddles and scintillating fibers. Also the dynamic range of CALET covers six order of magnitude which translates into a wide energy range from $1 \mathrm{MIP}$ to $1 \mathrm{PeV}$ shower energy [11]. In this paper, we present heavy nuclei components in the cosmic rays with the data obtained in the period from October 13, 2015 to December 31, 2018. Energy spectra of proton, carbon and oxygen are reported in other papers of this proceedings $[12,13]$.

\section{Instrument}

The CALET instrument consists of three detectors; CHarge Detector (CHD), IMaging Calorimeter (IMC) and Total AbSorption Calorimeter (TASC). CHD and IMC plays an important role for charge measurements and TASC for the energy measurement. CHD located at the top part is composed of two layers of 14 plastic scintillator paddles for measurement of the primary particle charge from $Z=1-40$. Each scintillator has dimensions of $32 \mathrm{~mm} \times 450 \mathrm{~mm} \times 10 \mathrm{~mm}$. IMC for the track reconstruction and charge measurements is a sampling calorimeter composed of $(X, Y) \times 8$ layers of scintillating fiber ( $\mathrm{SciFi}$ ) belts and 7 tungsten plates. Each layer of SciFi belts is made of $448 \mathrm{SciFis}$ with a $1 \mathrm{~mm}$ square cross section and $448 \mathrm{~mm}$ in length. The tungsten plates interleaved between the SciFi layers have thickness of $0.2 X_{0} \times 5$ layers and $1.0 X_{0} \times 2$ layers from top to bottom. TASC is a total absorption calorimeter made of 12 layers of PWO scintillator logs for energy measurement and discrimination of electromagnetic shower and hadronic shower. Each layer has 16 PWO $\operatorname{logs}$ and each $\log$ has dimensions of $19 \mathrm{~mm} \times 326 \mathrm{~mm} \times 20 \mathrm{~mm}$. The total thickness of the calorimeter is $30 X_{0}$ for electromagnetic particles or $1.3 \lambda_{I}$ for protons. The detector performance is evaluated by MC simulations and several beam tests at CERN-SPS using electrons, protons and nuclei [14-16]. 


\section{Data analysis}

We have analyzed flight data for 1,176 days from October 13, 2015 to December 31, 2018. The total observation live time is 23,698 hours. Figure 1 and 2 are candidates of magnesium and iron from flight data with $2.4 \mathrm{TeV}$ and $13.1 \mathrm{TeV}$ in observed energy respectively. Monte Carlo simulation data is produced by EPICS v9.22 and Cosmos 8.02 [21] with DPMJET-III [22] as a hadron interaction model. The MC data is smeared to reproduced flight data signals by considering fluctuations due to the pedestal rms noise, photo-statistics, and also quenching of light yield and saturation.

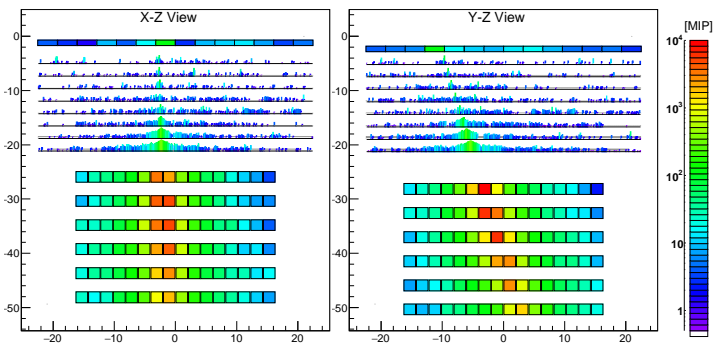

Figure 1: An example of magnesium candidate with $\Delta E_{\mathrm{TASC}}=2.4 \mathrm{TeV}$.

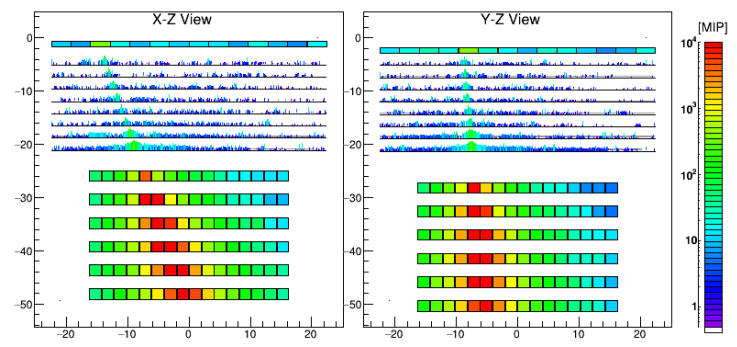

Figure 2: An example of iron candidate with $\Delta E_{\mathrm{TASC}}=13.1 \mathrm{TeV}$.

Event trigger Cosmic ray nuclei are detected by the high energy trigger [20] which requires a coincidence of two bottom layers of IMC and the top layer of TASC. The energy threshold is set to detect electrons above $10 \mathrm{GeV}$. In case of the light nuclei $(Z<10)$, only events creating the particle shower in the detector are detected because the trigger threshold is higher than the $d E / d x$ of penetrating events. On the other hand, the trigger efficiency of heavy nuclei is almost $100 \%$ because the $d E / d x$ of heavy nuclei is high enough to exceed the threshold. Therefore the shower event selection in offline analysis, which requires the signal in a layer among the upper four TASC layer to exceed the single MIP, is applied to measure their energy with the calorimeter. Figure 3 shows an example of the efficiency of the shower event selection for magnesium as a function of observed energy compared with MC simulation. Data and MC are in good agreement.

Detector calibration Energy calibration of each channel has been carried out by using penetrating cosmic-ray protons and helium nuclei $[15,17]$. Using the MIP events, the detector response including position, temperature and time dependence of the plastic scintillators and PWO logs have been well studied and equalized.

For charge identification, the non-linearity of CHD and IMC between detector response and deposit energy which is caused by scintillation quenching is obtained from the flight data. Figure 4 shows the Gaussian peaks in MIP unit of even charged particles as a function of $Z^{2}$ in the range of $31.6-51.2 \mathrm{GeV}$ of the deposit energy in TASC. It is fitted by a function based on the halo model $[18,19]$. Since the signal is affected by the $\delta$-ray production in the upstream materials, the parameters of CHD-X and CHD-Y are obtained independently. The energy dependence of the charge is corrected and the non-linearity of each IMC layer is also corrected with the same procedure of CHD. 


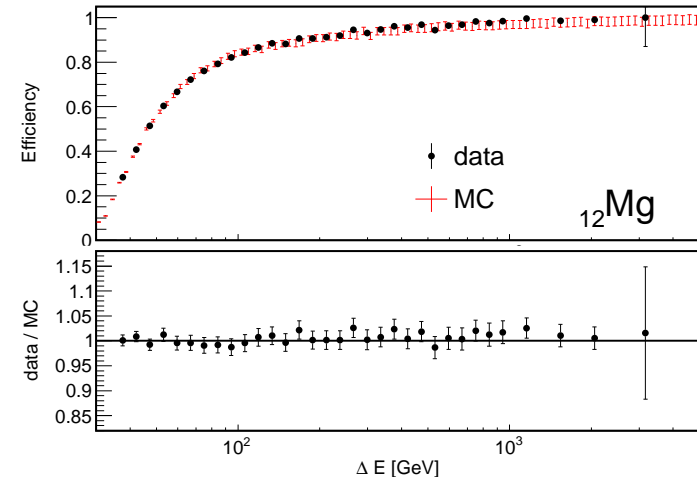

Figure 3: Efficiency of shower event selection for magnesium compared with MC.

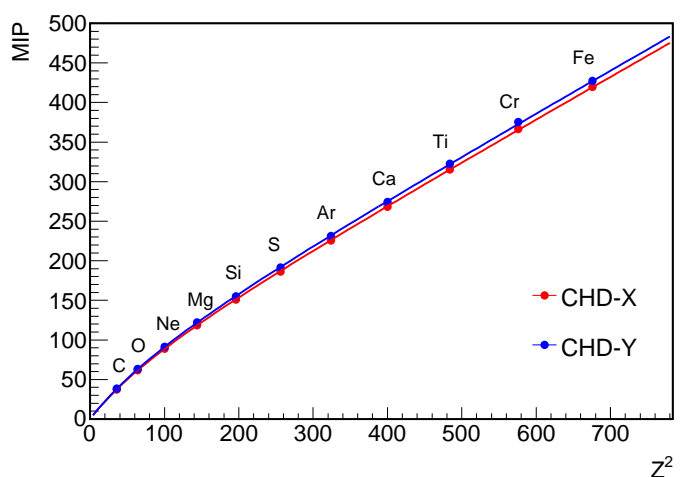

Figure 4: Detector response of CHD-X and CHD-Y with a fitting function.

Track reconstruction The accurate track reconstruction and estimation of the impact point at the CHD are essential for the charge identification. The shower axis is reconstructed by IMC signals. Although the nuclei create many shower particles in the IMC as shown in Fig. 1 and 2, which could be a large background for the track reconstruction, primary particle's signals are usually larger than the signals of the shower particles thanks to their large $d E / d x$ which is proportional to $Z^{2}$ disregarding the quenching effect. The shower axis is reconstructed by a least square fit using the maximum deposits per channel in the upper four IMC layers. The impact point at the CHD is estimated with an accuracy of $330 \mu \mathrm{m}$ for CHD-X and $300 \mu \mathrm{m}$ for CHD-Y with minor charge or energy dependence. Events with a fully reconstructed track are selected for the nuclei analysis in this paper, i.e., the track passes through the top surface of CHD and TASC and the bottom surface of TASC. The geometrical acceptance is $570.3 \mathrm{~cm}^{2} \mathrm{sr}$ which is calculated by means of MC simulation.

Charge identification Particle charge is identified on the basis of the $d E / d x$ measurements in $\mathrm{CHD}$ and IMC associated with the reconstructed track. Figure 5 shows the charge distribution of CHD with $\Delta E_{\text {TASC }}>10 \mathrm{GeV}$. The charge resolution is $0.18 e$ for carbon and $0.30 e$ for iron. The multiple $d E / d x$ measurements of IMC is also useful for light nuclei, while the IMC signal saturates for heavier charges. The charge resolution with upper four IMC layers is $0.19 e$ for carbon.

To maintain good charge resolution and remove background events, two selections are applied: the charge consistency among each CHD and IMC layer and the track width in IMC. These requirements are effective to remove events with mis-reconstructed track such as particles entering from the detector side and it is essential to remove background events interacting in the CHD. The requirement of charge consistency is that the difference of the charge between CHD-X and CHD-Y has to be less than $10 \%$. For light nuclei, charge consistency with IMC is also used and the requirement is a difference less than $15 \%$ of the truncated mean of the charge with 1st and 2nd IMC layers as well as that that with 3rd and 4th layers.

The track width of events interacting in the CHD is commonly wider than that of penetrating events due to the spread of the secondary particles, and the core signal of a nucleus passing through without an interaction shows larger signals than that of shower events. To exploit these characteristics, the difference of the sum of $7 \mathrm{SciFis}$ and the sum of $3 \mathrm{SciFis}$ normalized to the charge are 
used as track width parameter, which is effective not only for light nuclei but also for heavy nuclei.

Particle charge is identified by CHD within \pm 0.8 charge for iron and \pm 0.4 for the other species as individual elements in the analysis. The contamination from the other particles is estimated by MC events of elements with charge in $Z=1-28$. The MC event is reweighted with a factor to reproduce a signal power law spectrum with the index of -2.60 and all event selections are identical to the ones used for the flight data. The absolute value of each element in each observed energy bin is normalized to the charge distribution of CHD matching the flight data. The number of contaminant events is calculated by the integration of all contamination MC events. The total background is $3 \%$ for boron and less than a few percent for primary components with efficiencies of $25 \%$ for boron, $30-40 \%$ for carbon and oxygen and $50 \%$ for neon or heavier nuclei. The contaminants are subtracted before the energy unfolding as described in the following.

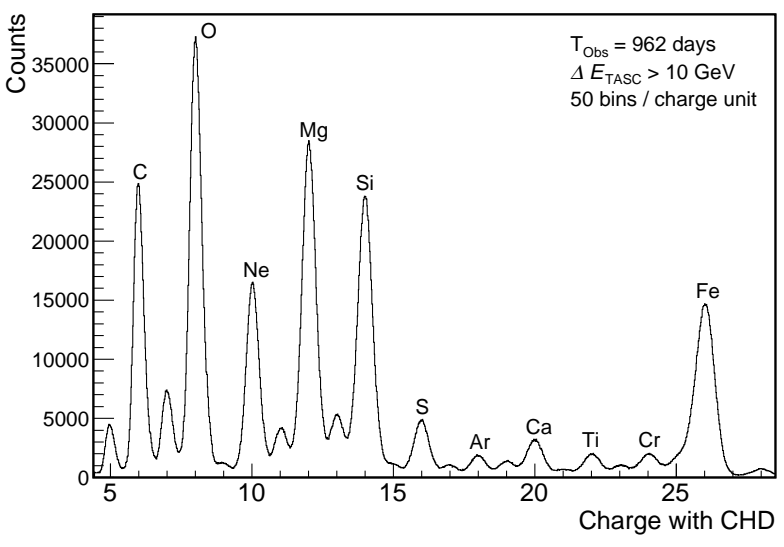

Figure 5: Charge distribution by combined CHD layers.

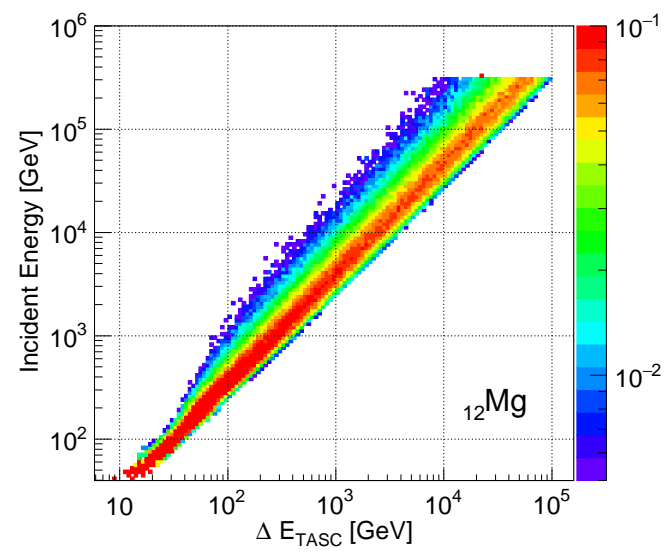

Figure 6: Response matrix of magnesium derived from MC simulations.

Energy measurement and unfolding The shower energy for each event is determined by the sum of energy deposits in the TASC. Since the energy leakage from the calorimeter is unavoidable for nuclei due to the characteristics of hadron induced showers and the finite detector resolution, an unfolding procedure for the derivation of the primary energy spectrum and to correct bin-to-bin migration is necessary. The iterative procedure based on Bayes's theorem [23] with the RooUnfold package [24] is applied with the response matrix of primary energy versus deposit energy, which is obtained from detailed MC simulations as shown in Fig. 6. For MC data the same event selection is applied as for flight data. Initial spectra are assumed as a single power law function with the index of -2.60 and two cycles of unfolding iteration are applied.

\section{Preliminary results}

Energy spectra of primary components Energy flux, $\Phi(E)$, is calculated as follows,

$$
\Phi(E)=\frac{N(E)}{\varepsilon S \Omega T \Delta E}
$$


where $N(E)$ is the number of events in the unfolded energy bin, $S \Omega$ is the geometrical acceptance, $T$ is the live time, $\Delta E$ is the width of energy bin and $\varepsilon$ is the total efficiency. Figure 7 shows preliminary results of energy spectra of neon, magnesium, silicon, sulfur, calcium and iron as a function of kinetic energy per particle with 1,176 days of operations. These spectra are comparable with previous observations $[5,6,25-29,31]$.

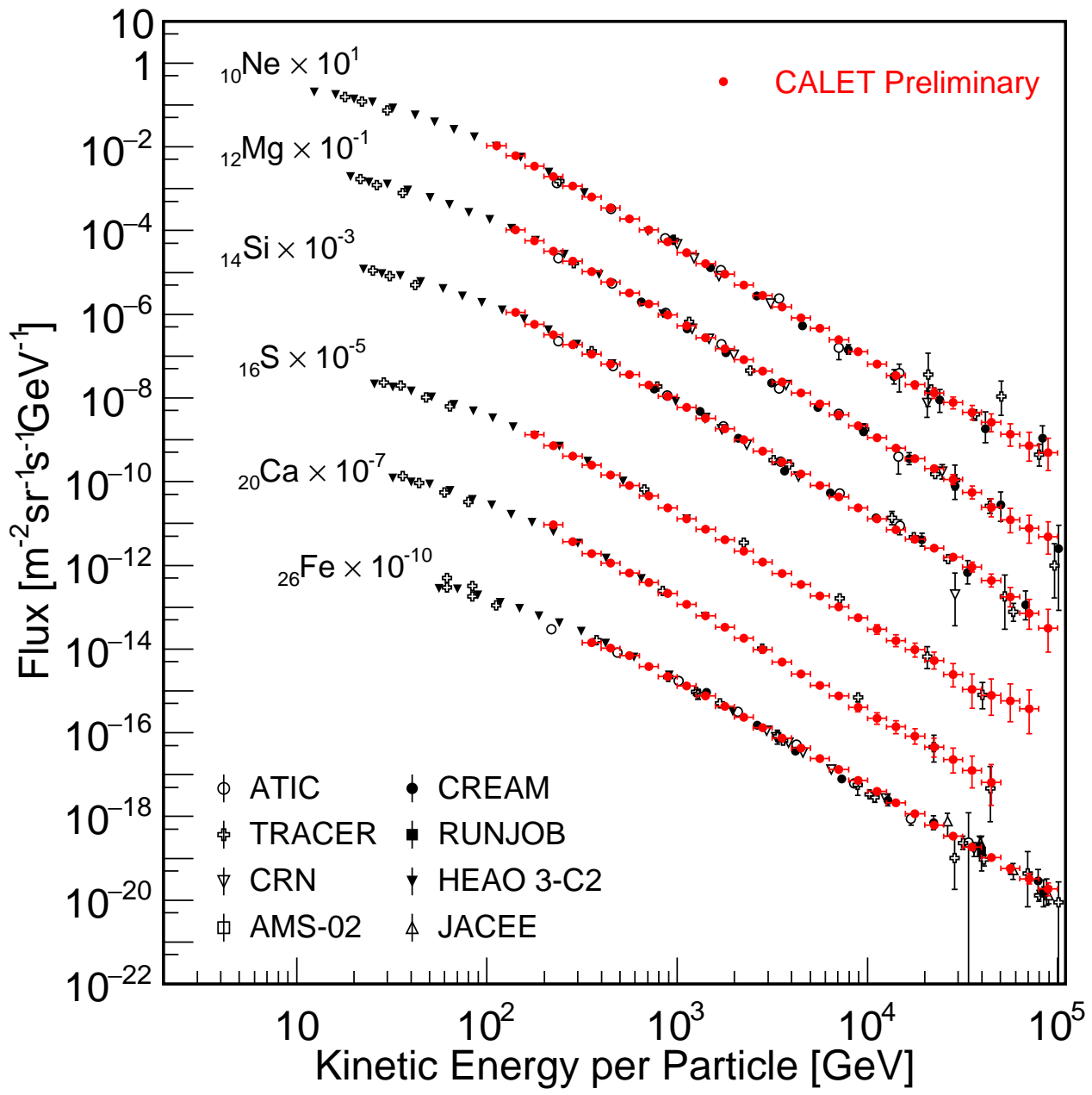

Figure 7: Preliminary results of energy spectra of heavy primary components of $\mathrm{Ne}, \mathrm{Mg}$, $\mathrm{Si}, \mathrm{S}, \mathrm{Ca}$ and $\mathrm{Fe}$ as a function of energy par particle compared with previous observations [5,6, 25-29, 31]. Error bars of CALET data represent the statistical uncertainty only.

Secondary-to-primary flux ratio Figure 8 shows a preliminary result of boron-to-carbon flux ratio with 1,176 days of operations. The isotopic composition is assumed as ${ }^{10} B:{ }^{11} B=3: 7$ based on the previous observation [32]. We have studied a number of systematic uncertainties including trigger efficiency, charge consistency cuts, charge selection window, energy correction with beam test, initial prior spectra of energy unfolding, response matrix of $\mathrm{MC}$ simulation and long-term stability. The sum in quadrature of statistical and systematic errors are shown in the gray band of 
the Fig. 8. The preliminary result of $\mathrm{B} / \mathrm{C}$ ratio with CALET in the energy range from $50 \mathrm{GeV} / \mathrm{n}$ to $200 \mathrm{GeV} / \mathrm{n}$ is in good agreement with previous observations [29,30,32-35].

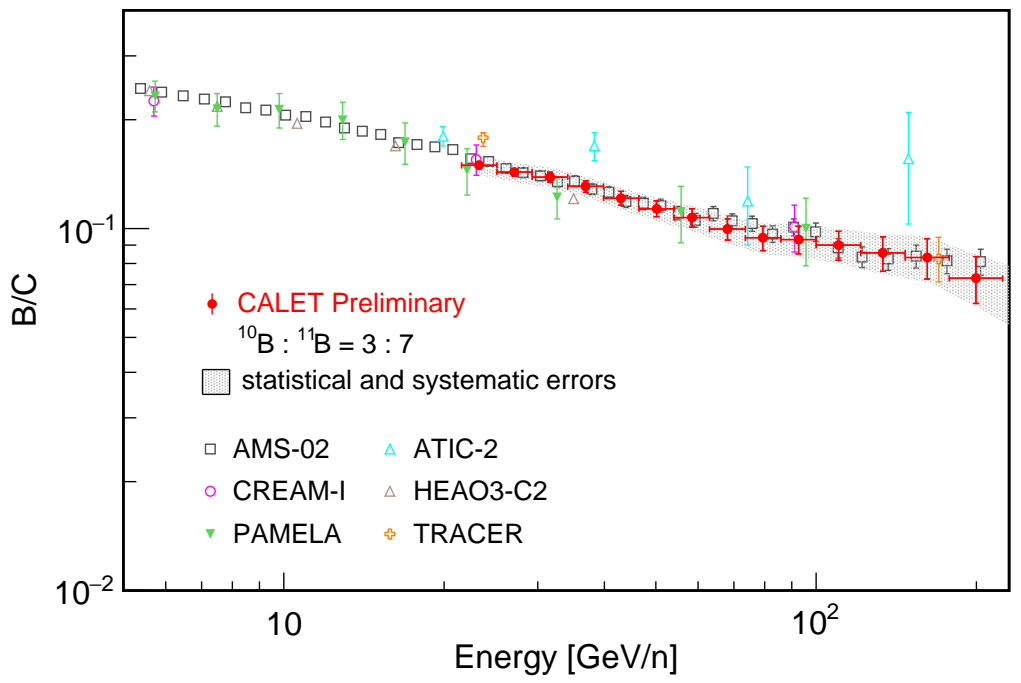

Figure 8: Preliminary result of boron-to-carbon flux ratio with CALET compared with previous observations [29,30,32-35].

\section{Conclusions}

The ability of CALET to measure heavy cosmic-ray nuclei has been successfully demonstrated and preliminary energy spectra have been derived for the primary cosmic ray elements up to $100 \mathrm{TeV}$ and boron-to-carbon ratio up to $200 \mathrm{GeV} / \mathrm{n}$ after 38 months of operation. The derived spectra illustrate the excellent capability of CALET to measure nuclei with high statistics in a wide energy range. We note that the event selection we have applied here is based on a preliminary analysis as compared to what can eventually be achieved with CALET. Further studies on an increased data set and detailed systematic study will increase the sensitivity to detailed spectral features, which may provide a key to solve questions about galactic cosmic-ray acceleration and propagation.

\section{References}

[1] O. Adriani et al., Science 322, 69 (2011)

[2] M. Aguilar et al., Phys. Rev. Lett. 114, 171103 (2015)

[3] O. Adriani et al., Phys. Rev. Lett. 122, 181102 (2019)

[4] M. Aguilar et al., Phys. Rev. Lett. 115, 211101 (2015)

[5] H. Ahn et al., Astrophys. J. 707, 14 (2011)

[6] M. Aguilar et al., Phys. Rev. Lett. 119, 251101 (2017) 
[7] S. Torii et al., PoS (ICRC2017) 1092

[8] Y. Asaoka et al., PoS (ICRC2019), inpress

[9] O. Adriani et al., Phys. Rev. Lett. 119, 181101 (2017)

[10] O. Adriani et al., Phys. Rev. Lett. 120, 261102 (2018)

[11] D. Ito et al., Proc. of 32nd ICRC 6, 375 (2011)

[12] P. S. Marrocchesi et al., PoS (ICRC2019), inpress

[13] P. Maestro et al., PoS (ICRC2019), inpress

[14] Y. Akaike et al., Proc. of 33rd ICRC, 0726 (2013)

[15] T. Niita et al., Adv. Sp. Res. 55, 9 (2015)

[16] Y. Akaike et al., PoS (ICRC2015) 613

[17] Y. Asaoka et al., Astropart. Phys. 91, 1 (2017)

[18] G. Tarlé et al., Astrophys. J. 230, 607 (1979)

[19] P.S. Marrocchesi et al., Nucl. Instrum. Meth. A 659, 477 (2011)

[20] Y. Asaoka et al., Astropart. Phys. 100, 29 (2018)

[21] K. Kasahara et al., Proc. of 24th ICRC 1, 399 (1995)

[22] S. Roesler et al., Proc. of Monte Carlo 71, 23 (2003)

[23] G. D'Agostini et al., Nucl. Instrum. Meth. A 362, 487 (1995)

[24] T. Adye, arXiv:1105.1160 (2011)

[25] A.D. Panov et al., Bull. Russian Acad. Sci. 73, 564 (2009)

[26] A. Obermeier et al., Astrophys. J. 724, 14 (2011)

[27] V.A. Derbina et al., Astrophys. J. 628, L41 (2005)

[28] D. Muller et al., Astrophys. J. 374, 356 (1991)

[29] J.J. Engelmann et al., Astron. Astrophys. 96, 233 (1990)

[30] O. Adriani et al., Astrophys. J. 791, 93 (2014)

[31] K. Asakimori et al., ApJ 502, 278 (1998)

[32] M. Aguilar et al., Phys. Rev. Lett. 117, 231102 (2016)

[33] H. Ahn et al., Astropart. Phys. 30, 133 (2008)

[34] A.D. Panov et al., Proc. of 30th ICRC 2, 3 (2008)

[35] A. Obermeier et al., Astropart. Phys. 752, 69 (2012) 